\title{
ORIGINAL ARTICLE \\ Positive and negative affect in individuals with spinal cord injuries
}

\author{
JE Salter ${ }^{1}$, SD Smith ${ }^{2}$ and KD Ethans ${ }^{1}$
}

Study design: Participants with spinal cord injuries (SCls) and healthy controls completed standardized questionnaires assessing depression level, positive and negative affect, and personality traits.

Objectives: To identify the specific characteristics of emotional experiences affected by spinal cord injury.

Setting: A Canadian rehabilitation center. Individuals with $\mathrm{SCls}$ were recruited from a list of patients who had volunteered to participate in studies being conducted by the $\mathrm{SCl}$ clinic. Healthy controls were recruited from the community, but tested in the $\mathrm{SCl}$ clinic.

Methods: Thirty-six individuals with complete (ASIA A) SCIs and 36 age-, gender- and education-matched controls participated in this study. SCl participants were classified as cervical (C1-C7), upper thoracic (T1-T5) or lower thoracic/upper lumbar (T6-L2). All participants completed the Beck Depression Inventory, the Positive and Negative Affect Schedules, the NEO Neuroticism Questionnaire, and the harm avoidance scale of the Tridimensional Personality Questionnaire. Data were analyzed using independent-samples $t$-tests (when contrasting $\mathrm{SCl}$ and controls) and analysis of variance (when comparing across $\mathrm{SCl}$ groups).

Results: Participants with SCls experienced significantly less positive affect than controls. The two groups did not differ in their experience of negative affect. Participants with SCls also reported greater levels of depression. Depression scores improved with an increasing number of years post injury.

Conclusion: Individuals with SCls are characterized by specific emotional dysfunction related to the experience of positive emotions, rather than a tendency to ruminate on negative emotions. The results suggest that these individuals would benefit from rehabilitation programs that include training in positive psychology.

Spinal Cord (2013) 51, 252-256; doi:10.1038/sc.2012.105; published online 11 September 2012

Keywords: emotion; spinal cord injury; positive affect; negative affect; depression

\section{INTRODUCTION}

The psychological consequences of spinal cord injury (SCI) are difficult to characterize, as the period immediately following the SCI is complicated by the confounding effects of medication, sensory deprivation, isolation and pain. ${ }^{1}$ However, a burgeoning literature has recently identified a number of emotional dysfunctions in these patients and has focused attention on the urgent need to address these issues during both acute and outpatient treatment. ${ }^{2}$ The majority of the existing studies of emotional impairments in individuals with SCIs have focused on depression, as it is the most common psychopathology following SCI. ${ }^{1}$ Indeed, the incidence rate in individuals with SCIs is much higher than that of healthy individuals ${ }^{3}$ with estimates ranging from $10-30 \%$ of this patient group. ${ }^{4,5}$ The prevalence of depression in individuals with SCIs is problematic, as it is related to subjective health, life satisfaction, and daily functioning abilities, and thus has a dramatic effect on the patients' ability to cope with their injury. ${ }^{6}$

Despite this emerging knowledge base, there are certain aspects of the emotional functioning of individuals with SCIs that require further elucidation. Notable among these is the effect that SCI has on an individual's ability to experience positive and negative emotions during daily life. As dysfunction of the autonomic nervous system is a common consequence of $\mathrm{SCI},{ }^{7}$ damage to the spinal cord could conceivably impair the transmission of bodily feelings or 'somatic markers' to the brain, thereby blunting subjective emotional experiences. ${ }^{8}$ Initial interview-based studies of SCI patients were consistent with this view, with patients reporting that emotions felt more cognitive and less visceral than before their injury. ${ }^{9}$ However, reduced emotionality was not reported in recent studies, ${ }^{10,11}$ thereby challenging the position that SCIs dampen all affective experiences. The goal of the current study was to assess both positive and negative affect in order to more precisely characterize the subjective emotional well-being of individuals with SCIs. On the basis of previous research, we hypothesized that individuals with SCI would show reduced levels of positive affect and higher levels of negative affect relative to healthy controls. To test this hypothesis, participants with SCIs and controls completed standardized questionnaires in order to identify the specific affective experiences that are, and are not, affected by SCIs.

${ }^{1}$ Department of Physical Medicine and Rehabilitation, University of Manitoba, Winnipeg, Manitoba, Canada and ${ }^{2}$ Department of Psychology, University of Winnipeg, Winnipeg, Manitoba, Canada

Correspondence: Dr JE Salter, Department of Physical Medicine and Rehabilitation, University of Manitoba, R 134800 Sherbrook Street, Winnipeg, Manitoba R3A 1M4, Canada. E-mail: umsalte2@cc.umanitoba.ca

Received 28 February 2012; revised 11 July 2012; accepted 24 July 2012; published online 11 September 2012 


\section{MATERIALS AND METHODS}

\section{Participants}

Participants consisted of 36 individuals with complete (American Spinal Cord Association-ASIA A) SCIs recruited from the Spinal Cord Injury Clinic at the Health Sciences Center in Winnipeg, Manitoba, Canada (see Table 1 for demographic characteristics). Fourteen of the individuals with SCIs had an injury between $\mathrm{C} 1$ and $\mathrm{C} 7$, nine had an upper thoracic injury between $\mathrm{T} 1$ and T5, and 13 had a lower thoracic or lumbar injury between T6 and L2. The thoracic lesions were divided as major sympathetic outflow is thought to originate above T6. These individuals were recruited from a clinic list of patients who had provided written consent to be contacted regarding research studies being conducted at the clinic. Participants were contacted by phone. After hearing a description of the study, participants were free to decide whether they wished to participate. Participants were specifically told that their decision would not negatively impact their care by the physicians and staff.

Thirty-six age-, education- and gender-matched healthy control participants were recruited from the Winnipeg community. Exclusion criteria included a history of neurological or psychiatric illness or the use of psychotropic drugs. Data from five control participants were removed due to a history of recreational drug use.

All participants provided informed consent prior to the commencement of this study. This project was reviewed and approved by the Bannatyne Campus Human Research Ethics Committee at the University of Manitoba and by the Senate Committee for Ethics in Human Research and Scholarship at the University of Winnipeg. Participants with SCIs and controls were both paid $\$ 20$ for participating in one 90-min session.

\section{Materials}

SCI and control participants completed four different questionnaires. All of the questionnaires were computerized using E-Prime software (Psychology Software Tools Inc., Pittsburgh, PA, USA) run on a Dell Latitude 620 laptop computer (Dell Canada Inc., North York, ON, Canada). The use of computerized questionnaires allowed the experimenter to use an external keyboard to enter all participants' responses, as some SCI participants had limited upper extremity mobility. This set-up also provided participants with a degree of privacy, as the experimenter could enter responses without viewing the questions on the monitor.

Assessments of emotional well-being. Subjective emotional well-being was assessed using two questionnaires. The Beck Depression Inventory $\mathrm{II}^{12}$ (BDI-II) is a 21-item measure of depression. On each question, participants select a response that is worth $0,1,2$ or 3 points. A total score of $0-13$ indicates minimal depression, 14-19 indicated mild depression, 20-28 indicates moderate depression and 29-63 indicates severe depression. The BDI-II has a test-retest reliability of $r=0.93$ and an internal consistency of $\alpha=0.91 .{ }^{12}$ Participants also completed the PANAS-X Scale, a variant of the Positive and Negative Affects Schedule; this measure has high test-retest reliability and internal consistency $(r=0.93 ; \alpha=0.83) .{ }^{13}$ This questionnaire consists of 60 words relating to an emotional (for example, cheerful, angry), physical (for example, tired, at ease) or socioemotional (for example, active, irritable) state. Participants indicate the degree to which their current feelings match each term using a 5-point scale ranging from 1 (very slightly or not at all) to 5 (extremely). The PANAS-X includes general emotional subscales measuring positive and negative affect. It also includes scales of basic negative emotions (fear, hostility, guilt and sadness), basic positive emotions (joviality, selfassurance and attentiveness) and other affective states (shyness, fatigue, serenity and surprise).

Assessments of personality traits. In order to control for the fact that innate personality differences might underlie any group differences found on assessments of emotional well-being, participants were also asked to complete two personality tests. The neuroticism scale of the NEO (Neuroticism, Extroversion and Openness-to-Experience Scale ${ }^{14}$ ) consists of 64 brief statements (for example, 'I often get angry at the way people treat me'). Participants are asked to indicate whether they agree or disagree with each statement using a 5-point Likert-style scale with 1 representing 'strongly disagree' and 5 representing 'strongly agree'. This scale has high reliability $(r=0.93)$ and internal consistency (Cronbach's alpha scores ranging from 0.89-0.93). ${ }^{14}$ The Harm Avoidance component of the Tridimensional Personality Questionnaire ${ }^{15}$ is a 34 -item measure of risk aversion $(r=0.81$, $\alpha=0.89$ ). Participants respond 'true or false' to each of 34 short statements (for example, 'I usually am confident that everything will go well, even in situations that worry most people'). These tests were selected because they have been shown to predict responses to emotional stimuli. ${ }^{16}$

\section{Procedure}

All participants provided informed written consent at the beginning of each test session. Participants then provided the experimenters with demographic information as well as with details about their past medical history and current medications. Following this, all individuals with SCIs underwent a 10-min physical examination with a physician to confirm the neurological level and impairment rating (ASIA A).

Participants then completed computerized versions of the NEO, Tridimensional Personality Questionnaire, Beck Depression Inventory and PANAS-X questionnaires. The order of administration was randomized across participants. After the completion of the final questionnaire, participants were told the purpose of the study and received remuneration.

\section{Data analysis}

Scores on each of the questionnaires were tabulated for all participants. In order to maximize statistical power, all participants with SCIs (regardless of the level of injury) were combined into a single SCI group for comparisons with healthy controls; these comparisons were conducted with independent-samples $t$-tests. In order to examine whether the level of SCI influenced responses, separate averages were calculated for each of the three participant groups with SCIs. Separate analysis of variance calculations were performed to compare all four groups (three SCI subgroups and controls). Finally, the results of the three SCI subgroups were compared in order to determine whether the level of SCIinfluenced performance.

\section{RESULTS}

\section{Assessments of emotional well-being}

The results of the BDI-II are depicted in Table 2. Consistent with previous research, the depression scores of the SCI patients were significantly elevated compared with those of healthy controls:

Table 1 The demographic details of the $\mathrm{SCl}$ participants and healthy controls

\begin{tabular}{|c|c|c|c|c|c|}
\hline & $C 1-C 7$ & T1-T5 & $T 6-L 2$ & SCl (average) & Controls \\
\hline Participants & 14 & 9 & 13 & 36 & 31 \\
\hline Gender (M:F) & 13:1 & $8: 1$ & 10:3 & $31: 5$ & $26: 5$ \\
\hline Age & $43.14(10.93)$ & $46.67(7.76)$ & $47.31(9.60)$ & $45.53(9.67)$ & $43.1(10.83)$ \\
\hline Education & $1.21(2.08)$ & $1.44(1.33)$ & $1.77(2.24)$ & 1.47 (1.95) & $2.58(2.26)$ \\
\hline
\end{tabular}


$\mathrm{t}(65)=2.293, P<0.03$. A regression analysis was performed to determine if scores on the BDI-II were predicted by the time since the SCI had occurred. Consistent with previous research, depression levels decreased as a function of time post-injury: $\mathrm{F}(1,35)=5.115$, $\mathrm{MSE}=51.992, P<0.03$. Age, gender and education-level did not predict depression scores (all F-values $<1$ ).

Analyses of the PANAS-X scores indicated that participants with SCIs experienced significantly less positive affect than controls: $\mathrm{t}(64)=2.033, P<0.05$ (see Figure 1). There was no difference in levels of negative affect $(\mathrm{t}<1)$. The positive- and negative-affect scores did not differ between SCI groups, nor was the analysis of variance comparing all four test groups significant (both $\mathrm{F}$-values $<1.50$ ). Additional comparisons were performed to examine the relationship

Table 2 Depicts the median depression, negative-affect, positiveaffect and personality scores of the $\mathrm{SCl}$ participants (collapsed across lesion level) and healthy controls

\begin{tabular}{|c|c|c|c|c|c|c|}
\hline & \multicolumn{2}{|c|}{$\mathrm{SCls}$} & \multicolumn{2}{|c|}{ Controls } & \multicolumn{2}{|c|}{ Statistics } \\
\hline & Median & $\begin{array}{c}\text { Confidence } \\
\text { intervals }\end{array}$ & Median & $\begin{array}{c}\text { Confidence } \\
\text { intervals }\end{array}$ & t-value & P-value \\
\hline BDI & 7.5 (12.0) & $7.0-12.3$ & $4.0(8.0)$ & $4.0-7.0$ & 2.293 & 0.025 \\
\hline NA & $1.8(0.8)$ & $1.6-2.0$ & $1.5(0.5)$ & $1.5-1.9$ & 0.956 & 0.343 \\
\hline PA & $3.40(0.8)$ & $3.0-3.45$ & $3.6(0.7)$ & $3.5-3.8$ & 2.033 & 0.046 \\
\hline $\mathrm{HA}$ & $55.0(14.0)$ & $52.6-58.4$ & $58.0(7.0)$ & $56.7-60.2$ & 1.722 & 0.090 \\
\hline NEO & $157.0(37.0)$ & 151.7-166.3 & $153.0(24.0)$ & $146.1-158.2$ & 1.536 & 0.129 \\
\hline
\end{tabular}

Abbreviations: BDI, Beck Depression Inventory; HA, harm avoidance; NA, PANAS negative affect; NEO, neuroticism; PA, PANAS positive affect; $\mathrm{SCl}$, spinal cord injury. Interquartile ranges are shown in parentheses.

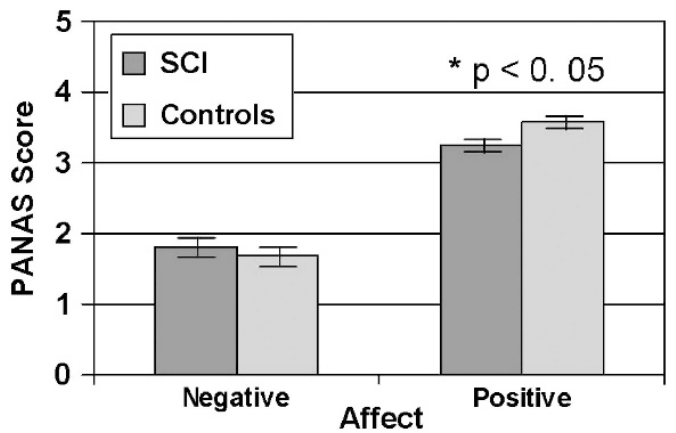

Figure 1 Scores for $\mathrm{SCl}$ participants and controls on the positive-affect and negative-affect categories of the PANAS-X questionnaire. Error bars represent standard error of the mean. between positive and negative affect, depression and demographic variables (see Table 3 ). Negative-affect scores were positively correlated with depression levels $(r=0.684, P<0.01)$, whereas positiveaffect scores were negatively correlated $(r=-0.464, P<0.01)$. Negative affect did not vary as a function of time post injury: $\mathrm{F}(1,35)=0.03, \mathrm{MSE}=0.008, P=0.863$. Positive affect appeared to increase as a function of time, although this trend was not significant: $\mathrm{F}(1,35)=2.03, \mathrm{MSE}=1.09, P=0.163$. Age, gender and educationlevel did not predict PANAS-X scores (both F-values $<1.90$ ).

Separate contrasts were performed for each of the 11 subscales of the PANAS-X described above (see Table 4). SCI participants showed greater levels of reported hostility than did controls: $\mathrm{t}(65)=2.379$, $P<0.025$. However, this effect was not significant when corrected for multiple comparisons. The data also included trends suggesting that the SCI groups may experience greater levels of sadness $(\mathrm{t}(65)=1.958$, $P=0.055)$ and lower levels of joviality $(\mathrm{t}(65)=1.738, P=0.087)$ than do healthy controls. No other between-groups comparisons approached statistical significance.

\section{Assessments of personality traits}

The measures of personality traits showed few differences between SCI participants and healthy controls (see Table 2). The neuroticism scores of the SCI group were greater than those of controls; however, this difference was not statistically significant: $\mathrm{t}(65)=1.536$, $P=0.129$. NEO scores did not predict BDI-II or PANAS-X scores (all F-values $<1.10$ ). Harm avoidance scores did differ between the groups, although the results were only marginally significant $(\mathrm{t}(65)=1.722, P=0.090)$. SCI participants were less harm-avoidant than were controls; regression analyses found that this measure did not predict BDI-II or PANAS-X scores (all F-values $<1.30$ ).

\section{Level of spinal cord injury}

Separate analyses were performed to examine whether the level of SCI influenced responses on the different experimental measures. The depression levels of the cervical SCI group were significantly greater than those of controls $(P<0.02)$. However, no difference existed between the different SCI groups $(\mathrm{F}<0.60)$. Additionally, no differences existed between SCI groups for positive or negative-affect scores; controlling for personality variables did not alter these results. Thus, it appears that while SCIs can alter emotionality, this effect is not influenced by the level of the injury.

\section{DISCUSSION}

The primary goal of the current research was to identify specific emotional impairments associated with SCIs. Consistent with our hypotheses, the results indicate that individuals with SCIs experience

Table 3 Correlations between variables for both $\mathrm{SCl}$ and control participants

\begin{tabular}{|c|c|c|c|c|c|c|c|c|}
\hline & $B D I$ & $N A$ & $P A$ & $H A$ & NEO & Age & $E d$ & Yrs SCI \\
\hline BDI & - & $0.665^{* *}$ & $-0.482^{* *}$ & $-0.724^{* *}$ & $0.782^{* *}$ & -0.185 & -0.274 & $-0.362^{*}$ \\
\hline NA & $0.734^{* *}$ & - & -0.199 & $-0.519^{* *}$ & $0.614^{* *}$ & -0.169 & -0.244 & -0.030 \\
\hline PA & -0.308 & -0.081 & - & $0.480^{* *}$ & $-0.518^{* *}$ & 0.232 & -0.050 & 0.241 \\
\hline $\mathrm{HA}$ & -0.352 & $-0.440^{*}$ & 0.143 & - & $-0.760^{* *}$ & -0.192 & -0.090 & -0.001 \\
\hline NEO & $0.542^{* *}$ & $0.577^{* *}$ & $-0.400^{*}$ & $-0.629^{* *}$ & - & -0.107 & -0.292 & -0.193 \\
\hline Age & -0.012 & -0.306 & -0.274 & 0.212 & -0.176 & - & 0.314 & $0.573^{* *}$ \\
\hline Ed & -0.311 & -0.155 & -0.083 & 0.242 & -0.057 & -0.166 & - & $0.439^{* *}$ \\
\hline Yrs SCl & - & - & - & - & - & - & - & - \\
\hline
\end{tabular}

Abbreviations: BDI, Beck Depression Inventory; Ed, education level; HA, harm avoidance; NA, negative affect; NEO, neuroticism; PA, positive affect; Yrs SCI, years since spinal cord injury. $\mathrm{SCl}$ participants' data are depicted on the top-right of the table (horizontal); control data are shown on the bottom-left of the table (vertical). ${ }^{*} P<0.05$; ${ }^{* *} P<0.01$. 
Table 4 Median for the 11 subscales of the PANAS-X

\begin{tabular}{lcc}
\hline PANAS Scale & SCls & Controls \\
\hline Attentiveness & $3.75(1.00)$ & $3.75(1.00)$ \\
Fatigue & $2.50(1.00)$ & $2.50(1.00)$ \\
Fear & $1.67(1.00)$ & $1.50(0.83)$ \\
Guilt & $1.50(1.00)$ & $1.50(0.67)$ \\
Hostility & $2.00(0.83)$ & $1.50(0.50)$ \\
Joviality & $3.25(0.75)$ & $3.50(0.88)$ \\
Sadness & $1.80(1.00)$ & $1.40(1.00)$ \\
Self assurance & $3.00(1.20)$ & $3.33(1.00)$ \\
Serenity & $3.67(1.00)$ & $3.33(1.33)$ \\
Shyness & $1.75(1.00)$ & $1.52(0.75)$ \\
Surprise & $2.33(1.33)$ & $2.33(1.00)$
\end{tabular}

Abbreviations: PANAS, Positive and Negative Affects Schedule, SCI, spinal cord injury. Interquartile ranges are listed in parentheses.

reduced levels of positive affect compared with healthy controls. However, our hypothesis that SCI participants would show increased negative affect was not confirmed, despite the fact that these individuals had larger BDI scores than controls. These results suggest that individuals with SCIs experience a blunting of affect, possibly due to attenuated feedback from the autonomic nervous system. ${ }^{7}$ Additionally, these data indicate that as the time since SCI increases, depression levels decrease. Given the trend of positive affect increasing over the course of treatment, we speculate that these improvements in the subjective well-being of individuals with SCIs are due to improved levels of positive affect rather than reductions in negative affect.

The level of SCI did not influence the levels of positive or negative affect experienced by the participants. These results contradict Hohmann's ${ }^{9}$ early interview-based study with SCI patients, which indicated that individuals with upper spinal cord lesions experienced colder, less visceral emotions than did patients with lower spinal cord lesions. However, our results are consistent with more recent work demonstrating that SCIs did not influence performance on a test of emotional decision-making (the Iowa Gambling Task). ${ }^{17}$ One explanation for the difference between Hohmann's qualitative report and our questionnaire-based study is that his patients' reports were vague and open to interpretation. A more likely explanation is that his patient group was depressed. Many of the patients with SCIs in his study were recent Vietnam War veterans recovering from injuries in Veterans Administration hospitals. Given that our data indicate that depression scores decrease as a function of recovery time, it is possible that the patient reports may have become less negative as well. Future research could address this issue by combining interview- and questionnaire-based methodologies in a longitudinal study of SCI participants. ${ }^{18}$

Although the current results provide novel information about the affective experiences of individuals with SCIs, this study does have a number of limitations. The sample size of 36 was suitable for simple contrasts between SCI and healthy control groups; however, there was insufficient statistical power for contrasts between the different SCI groups. Another limitation is the heterogeneity of the SCI population. Some of the SCI participants were injured as a result of risk-taking behavior (for example, driving or diving while under the influence) while others were the result of unfortunate circumstances (for example, a pedestrian who was hit by a car). Although the SCI participants provided verbal descriptions of the cause of their injury, it was not always possible to verify details to a degree that would allow us to incorporate 'cause of injury' into statistical analyses. Future research should attempt to include such information into analyses of the emotional effects of SCIs in order to determine if perceived responsibility for the SCI (self-caused vs other-caused) influences levels of depression as well as positive and negative affect. Finally, although an effort was made to control for the effect of personality variables that have been shown to influence emotionality, it is possible that traits other than neuroticism and harm avoidance could affect the results. Future studies should include a wider array of personality assessments to rule out such possibilities.

The results of the current study suggest that existing rehabilitation regimens for individuals with SCIs should be augmented in specific ways. Although focusing on alleviating symptoms of depression is critical for a patient's recovery, reducing depression is only one component of the challenges faced by medical teams. The fact that SCI participants experienced less positive affect is problematic, as these emotions promote subjective well-being and life satisfaction, and also increase emotional resilience in the face of everyday challenges. ${ }^{19}$ Indeed, positive affect has been linked with improved life satisfaction following SCIs. ${ }^{20}$ Therefore, the incorporation of positive psychology techniques such as loving-kindness meditation, a focus on self-efficacy, and the promotion of positive social and familial relationships, into counseling sessions for people with SCIs would likely prove beneficial.

\section{DATA ARCHIVING}

There were no data to deposit.

\section{CONFLICT OF INTEREST}

The authors declare no conflict of interest.

\section{ACKNOWLEDGEMENTS}

The authors wish to thank Michelle Di Nella, Stephanie Villeneuve and Michelle Crease for research assistance, and Tracy Olafson for assistance with patient recruitment. The authors also wish to thank all of the participants in this study for their time and conscientiousness. This study was sponsored by the Natural Sciences and Engineering Research Council (NSERC) of Canada.

1 North NT. The psychological effects of spinal cord injury: a review. Spinal Cord 1999; 37: 671-679.

2 Craig A, Tran Y, Middleton J. Psychological morbidity and spinal cord injury: a systematic review. Spinal Cord 2009; 47: 108-114.

3 Fann JR, Bombardier CH, Richards JS, Tate DG, Wilson CS, Temkin N. Depression after spinal cord injury: comorbidities, mental health service use, and adequacy of treatment. Arch Phys Med Rehabil 2011; 92: 352-360.

4 Hoffman JM, Bombardier CH, Graves DE, Kalpakjian CZ, Krause JS. A Iongitudinal study of depression from 1 to 5 years after spinal cord injury. Arch Phys Med Rehab 2011; 92: 411-418.

5 Saunders LL, Krause JS, Focht KL. A longitudinal study of depression in survivors of spinal cord injury. Spinal Cord 2012; 50: 72-77.

6 Bombardier CH, Richards JS, Krause JS, Tulsky D. Symptoms of major depression in people with spinal cord injury: implications for screening. Arch Phys Med Rehabil 2004; 85: 1749-1756.

7 Alexander MS, Biering-Sorensen F, Bodner D, Brackett NL, Cardenas D, Charlifue S et al. International standards to document remaining autonomic function after spinal cord injury. Spinal Cord 2009; 47: 36-43.

8 Damasio AR. Descartes' Error. Papermac/Macmillan: London, 1994.

9 Hohmann GW. Some effects of spinal cord lesions on experienced emotional feelings. Psychophys 1966; 3: 143-155.

10 Cobos P, Sánchez M, Garcia C, Nieves VM, Vila J. Revisiting the James versus Cannon debate on emotion: startle and autonomic modulation in patients with spinal cord injuries. Biol Psych 2002; 61: 251-269.

11 Deady DK, North NT, Allan D, Law Smith MJ, O'Carroll RE. Examining the effect of spinal cord injury on emotional awareness, expressivity and memory for emotional material. Psych Health Med 2010; 15: 406-415. 
12 Beck AT, Steer RA, Brown GK. Manual for Beck Depression Inventory // (BDI-II) Psychology Corporation: San Antonio, TX, 1996.

13 Watson D, Clark LA, Tellegen A. Development and validation of brief measures of. positive and negative affect: the PANAS scales. J Pers Soc Psych 1988; 54 1063-1070.

14 McCrae RR, Costa Jr PT. NEO Inventories: Professional manual. Psychological Assessment Resources Inc: Lutz, FL, 2010.

15 Cloninger CR, Przybeck TR, Svrakic DM. The tridimensional personality questionnaire: normative data. Psych Rep 1991; 69: 1047-1057.

16 Canli T. Functional brain mapping of extraversion and neuroticism: learning from individual differences in emotion processing. J Pers 2004; 72: 1105-1132.
17 North NT, O'Carroll RE. Decision making in patients with spinal cord damage: afferent feedback and the somatic marker hypothesis. Neuropsychologia 2001; 39: 521-524.

18 Pollard C, Kennedy P. A longitudinal analysis of emotional impact, coping strategies, and post-traumatic psychological growth following spinal cord injury: a 10-year review. Br J Health Psych 2007; 12: 347-362.

19 Quale AJ, Schanke AK. Resilience in the face of coping with a severe physical injury: a study of trajectories of adjustment in a rehabilitation setting. Rehabil Psychol 2010; 55: $12-22$.

20 Kortte KB, Gilbert M, Gorman P, Wegener ST. Positive psychological variables in the prediction of life satisfaction after spinal cord injury. Rehabil Psychol 2010; 55: 40-47. 